\title{
Do Tumor Characteristics and Pre-Transplant Locoregional Therapy Predict Survival after OLT in Patients with Hepatocellular Carcinoma?
}

\author{
Mohamed Kohla $^{1,2,3 *}$, Richard Shaw ${ }^{3}$, Garret Hisatake ${ }^{2}$, Robert Osorio ${ }^{2}$, Maurizio Bonacini ${ }^{2}$ \\ ${ }^{1}$ Department of Hepatology, National Liver Institute, Menoufiya University, Shebeen El-Kom, Egypt; ${ }^{2}$ Department of Transplantation, \\ California Pacific Medical Center, San Francisco, USA; ${ }^{3}$ Research Institute, California Pacific Medical Center, San Francisco, USA. \\ Email: *dr_mohamedsamy@yahoo.com
}

Received October $30^{\text {th }}, 2013$; revised November $19^{\text {th }}, 2013$; accepted December $15^{\text {th }}, 2013$

Copyright (C) 2013 Mohamed Kohla et al. This is an open access article distributed under the Creative Commons Attribution License, which permits unrestricted use, distribution, and reproduction in any medium, provided the original work is properly cited. In accordance of the Creative Commons Attribution License all Copyrights (C) 2013 are reserved for SCIRP and the owner of the intellectual property Mohamed Kohla et al. All Copyright (C) 2013 are guarded by law and by SCIRP as a guardian.

\begin{abstract}
HCC prognosis after OLT is associated with criteria related to the number and size. However, the degree of differentiation and efficacy of locoregional therapies may also influence outcome. Aim: Characterize patients with and without HCC and compare outcomes according to tumor characteristics. Methods: Retrospective query of an electronic medical record of 328 patients transplanted at California Pacific Medical Center (CPMC) in 2001-2007. HCC was defined by pre-OLT listing data as well as the finding of a tumor consistent with HCC at liver explant. Milan and UCSF criteria were applied to the lesions as described by pathology upon explant examination. Results: 328 patients were evaluated, with 109 liver malignancies, 103 females $(26(25 \%)$ HCC) and 225 males $(83(37 \%)$ HCC $p=0.04)$. HCC patients were older $(56 \pm 7.2 \mathrm{yr})$ than non HCC patients $(51 \pm 9.2, p<0.001)$. The age of the donor and cold ischemia time was not different in the 2 groups. Survival was shorter in HCC (mean $984 \pm 599$ days) vs. non HCC (1103 \pm 642 ) but not statistically significant $(p=0.10)$. Kaplan Meier survivals were superposable when comparing patients with or without malignancy and when patients with low $(\leq 22)$ vs. high MELD $(>22)$ were compared. Survival curves in patients that fulfilled Milan vs. UCSF criteria were identical. However, more patients outside Milan died of metastatic disease (5/6, $83 \%)$ vs. within Milan $(6 / 14,43 \%, p=0.01)$. Cox proportional hazards regression showed that MELD, but not malignancy, differentiation or necrosis, was associated with mortality; HR $=6 \%(95 \%$ C.I. $1 \%-10 \%)$ per additional MELD point $(p=0.02) .69$ pts had TACE pre-OLT, 17 had RFA \pm any other modality. There was no difference in survivals in pts who received any locoregional therapy vs. those who did not $(p=0.5)$. Deaths occurred in $20(18 \%)$ HCC vs. 43 $(19 \%)$ non HCC pts. Causes of mortality were different: of $20 \mathrm{HCC}$ patients, 11(55\%) died of HCC/metastatic disease vs. $2(5 \%)$ in 41 non HCC deaths $(p<0.0001)$. Conclusion: In our cohort, survival of HCC patients was comparable to that of non HCC patients. However, mortality from metastatic disease was higher, particularly in those outside Milan. Overall mortality was associated with higher MELD scores, but not with tumor necrosis, the degree of differentiation at explant or locoregional therapy.
\end{abstract}

Keywords: Tumor Characteristics; Locoregional Therapy; Hepatolcellular Carcinoma; Liver Transplantation; OLT; Survival

\section{Introduction}

Hepatocellualr carcinoma is the most common primary malignant tumor of the liver. Traditionally, the primary therapeutic modality for HCC has been surgical excision [1]. Optimal candidates for surgical resection show a

"Corresponding author. single lesion less than $5 \mathrm{~cm}$ in size, with no complications of end-stage liver disease and no significant portal hypertension (portal pressure gradient less than 10 $\mathrm{mmHg}$ ). Nevertheless, after 5 years there are significant recurrence rates $(70 \%)$ in $\mathrm{HCC}$ patients after surgical resection, and 5-year survival rate is 30\% [2-4]. Regeneration after resection, may elicit metachronous tumors 
(a theoretical concern, deserving attention). Efforts to prevent tumor recurrence have involved administration of retinoids and intra-arterial $\mathrm{I}^{131}$ labeled lipiodol $[5,6]$. In Western countries, where liver cancer typically develops in the setting of well-established cirrhosis, fewer than 5\% of patients are ideal candidates for hepatic resection [7], on the other hand, in Asian countries, the applicability of resection is higher, reflecting a less advanced liver disease related to chronic hepatitis B virus infection [8-10]. Collectively, no more than $25 \%$ of patients are candidates for surgical resection due to tumor size or location, multifocal disease, and poor hepatic reserve. In cirrhotic cases, OLT represents the only chance for curative therapy [1], because OLT has been claimed to simultaneously cure the malignant disease and replace the premalignant cirrhotic liver.

Early series of OLT for HCC yielded poor outcomes [3,11-20].

In those series, 3- and 5-year survival after OLT ranged $15 \%-67 \%$ and $15 \%-48 \%$, respectively. These inferior results reflected the inclusion of patients with advanced HCC. Subsequently, patients with confined HCC (solitary lesion $\leq 5 \mathrm{~cm}$ or $\leq 3$ lesions with diameter $\leq 3 \mathrm{~cm}$ ), no major vessel invasion and no extra hepatic involvement and Milan criteria, were reported to show an excellent long-term outcome with a 5 -year survival rate of $70 \%$ and a recurrence rate below $15 \%$ [21]. Based on pathologic review, modestly expanded selection criteria (solitary lesion $\leq 6.5 \mathrm{~cm}$, or $\leq 3$ lesions with the largest one $\leq 4.5 \mathrm{~cm}$ and a total tumor diameter $\leq 8 \mathrm{~cm}$ ), UCSF criteria were suggested to offer an excellent outcome with a 1 -year and 5-year survival rates of $90 \%$ and $75.2 \%$ respectively [22]. In clinical practice, however, the Milan criteria based on pre-OLT radiological findings, could be more useful and a more widely accepted selection criteria than the UCSF criteria based on post-OLT pathologic findings [23]. Generally, authors report overall patient survival rates of $35 \%$ to $58 \%$ at 5 years follow-up [3,13-16] with HCC recurrence associated with the poorest survival rates. In the pre-1990 era, HCC recurrence following OLT was reported as high as $84 \%$ [17]. The most important factors that have been described affecting OLT survival in patients with HCC include: tumor size, vascular invasion [18], degree of tumor differentiation [19], extra hepatic disease, and lymph node metastases [2]. Compared with the results before 1990, OLT in selected patients with HCC has seen significant improvements in patient and graft survival [20].

In order to decrease the waitlist dropout, various treatment modalities including resection, radiofrequency ablation (RFA), percutaneous ethanol injection (PEI), and transarterial chemoembolization (TACE) have been used to prevent HCC progression. It remains uncertain whether excellent outcomes can be obtained in HCC patients who previously underwent locoregional treatments (down staged) and met the Milan criteria at the time of OLT [23].

Taking into consideration the high dropout rate for patients with HCC awaiting OLT [24], an adjustment to the MELD score was implemented to give greater priority for organ allocation for patients with a solitary tumor of $2 \mathrm{~cm}$ or greater and those with two or three lesions, each not exceeding $3 \mathrm{~cm}$ (UNOS stage II criteria) [24]. These patients are assigned a MELD score of 29, equivalent to a 30\% 3-month mortality rate, and also are entitled to an additional increase in MELD score by 2 points for every 3 months on the waiting list without exceeding UNOS stage II criteria [24].

By reducing the waiting time for OLT for patients with HCC, this new scheme of organ allocation also may potentially justify a modest expansion of tumor size criteria OLT [25], and may improve intention-to-treat survival for HCC.

Tumor recurrence post transplant might be related to higher doses of immunosuppressives, and the latter are known to represent a significant risk factor for tumor growth, as shown in some experimental and clinical studies [26-28], in one of which [29], tumor recurrence was related to the dosage of the immunosuppression given in the first postoperative year when most of the recurrences developed. A high dosage of cyclosporin administered during postoperative months 3 to 12 was significantly related to a low recurrence-free survival. This observation suggested that the clinical studies carried out in the early 1990s, when the criteria for transplantation of heaptocellular carcinoma were introduced, and might have been influenced by immunosuppressive regimen adopted at that time [29].

\section{Aim of the Work}

To study the impact of hepatocellular carcinoma, preOLT locoregional therapy, and tumor characteristics on the outcome after OLT in patients who received a liver transplant (OLT) at California Pacific Medical Center.

\section{Methods}

A retrospective query of the electronic medical records at California Pacific Medical Center (OTTR), San Francisco, USA, was run for patients transplanted from 2001 till 2007.

Factors affecting the outcome after liver transplantation were studied.

Dependent variable

- Patient survival (time from OLT to death)

Independent variables

Donor variables:

Donor information was obtained from the United Net- 
work for Organ Sharing (UNOS) database.

- Donor race.

- Donor age.

- Donor gender.

- Donor HB core Ab status.

- Donor CMV status.

- Donor BMI.

- Cold ischemia time.

- Warm ischemia time. Recipient variables:

- Recipient race.

- Recipient age.

- Recipient gender.

- Etiology of liver disease.

- Presence of HCC.

- MELD at OLT.

- Bilirubin at OLT.

- Albumin at OLT.

- Sodium at OLT.

- Hemoglobin at OLT.

- Alcohol, assessed by social worker, Abuse/dependence vs. not.

- Diabetes pre-OLT and or post-OLT, HbAlc was evaluated just pre-OLT and at last post-OLT follow up.

- Number of immunosuppressive drugs at 1 year post transplant and at the last follows up.

- Estimated iron at explant pathology (0, trace, $1-4+)$.

- HCC by native liver pathology.

- HCC subcategories (number of nodules, largest nodule in $\mathrm{cm}$, lobar involvement, differentiation, vascular invasion, metastases).

- Type of pre-OLT locoregional therapy for HCC (TACE, RFA, resection, PEI, or combination).

- Effect of locoregional therapy for HCC in terms of necrosis at explant.

\section{Statistical Analysis}

Data were analyzed using the statistical package SPSS version 15 distributed by SPSS incorporator (SPSS Inc., v.15, Chicago, IL).

The following tests were run:

1) Univariate analysis, log rank test.

2) Cox Regression model using variables with $p<0.2$.

3) Kaplan Meier curves for actuarial survival.

\section{Results}

Statistical analysis was run on 328 patients who have been transplanted from the beginning of 2001 till the end of April 2007; the aim of this analysis was study HCC in particular.

\subsection{HCC and Overall Survival}

Out of 328 patients included in this analysis, 219 did not have HCC while 109 had HCC.

Figure 1 shows a Kaplan Meier survival analysis comparing the actuarial survival in patients with HCC (green legend) versus those without HCC (blue legend); the $p$ value by Wilcoxon test was 0.445 , which was statistically insignificant.

\subsection{Milan Criteria for HCC}

Patients were categorized into 3 groups for this analysis: First group: 219 patients without HCC (blue legend).

Second group: 96 patients within Milan criteria (green legend).

Third group: 7 patients outside Milan criteria (yellow legend).

N.B. 6 patients had incidental HCC on explants.

Figure 2 shows a Kaplan Meier survival analysis comparing the actuarial survival in the 3 groups; the $p$ value by Wilcoxon test was 0.549 , which was statistically insignificant.

\subsection{Pre-OLT Locoregional Therapy}

Patients were categorized into 4 groups according to type of pre-OLT locoregional therapy.

First group: 234 patients who did not receive any pre-OLT therapy (non-HCC or incidental, HCC who did not receive treatment, marked with blue legend).

Second group: 69 patients who received transarterial chemoembolization (TACE) alone pre-OLT (green legend).

Third group: 17 patients who received radiofrequency ablation pre-OLT (alone or in combination with others, marked with yellow legend).

Fourth group: 8 patients who received any other preOLT locoregional therapy (percutaneous ethanol injection, resection, etc, marked with purple legend).

Figure 3 shows a Kaplan Meier survival analysis comparing the actuarial survival in the 4 groups, the $p$ value by Wilcoxon test was 0.501 , which was statistically insignificant.

\subsection{Degree of HCC Differentiation at Explant Pathology}

Currently, all OLT recipients at CPMC have their native livers pathologically examined, it was assumed that HCC patients with well differentiated HCC may have a better survival than patients with moderately-poorly differentiated.

This analysis was run on 3 groups:

First group: 247 patients with no evidence of $\mathrm{HCC}$ at explants (blue legend).

Second group: 49 patients with well differentiated HCC (green legend). 


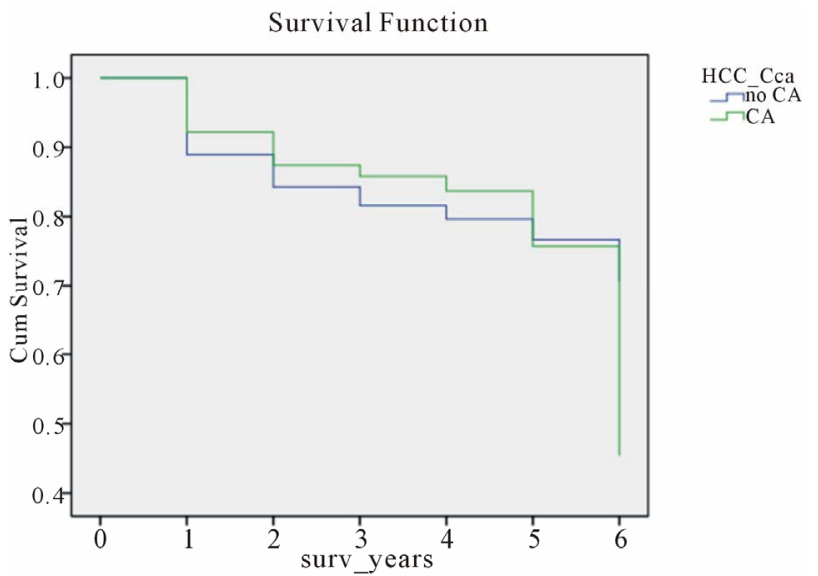

Figure 1. Kaplan Meier survival analysis comparing the actuarial survival in patients with HCC versus those without HCC.

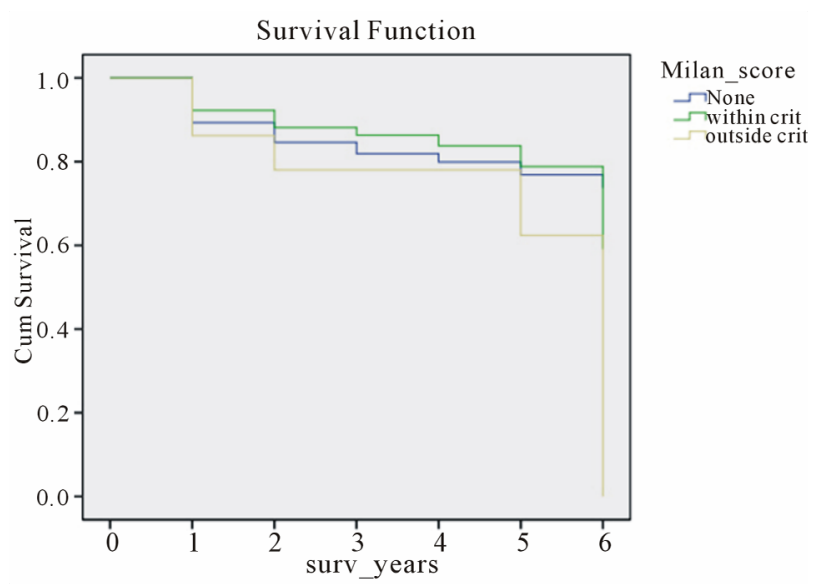

Figure 2. Kaplan Meier survival analysis comparing the actuarial survival according to Milan criteria (without HCC, within Milan and outside Milan criteria).
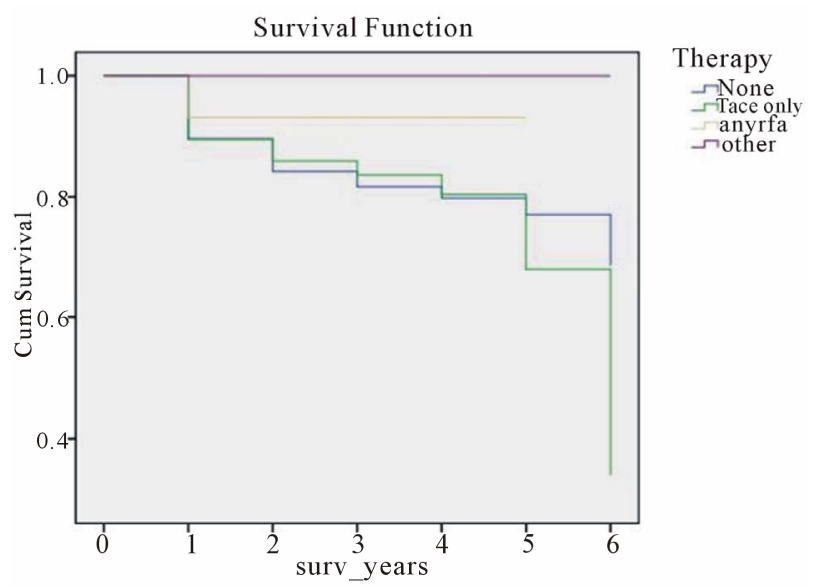

Figure 3. Kaplan Meier survival analysis comparing the actuarial survival according to the type of locoreginal therapy (non HCC, TACE only, any RFA and other types of therapy).
Third group: 32 patients with moderately-poorly differentiated HCC (yellow legend).

Figure 4 shows a Kaplan Meier survival analysis comparing the actuarial survival in the 3 groups, the $p$ value by Wilcoxon test was 0.178 , which was statistically insignificant.

However, when comparing each 2 groups to each other, those with well differentiated HCC showed a trend of better survival than those with moderately-poorly differentiated HCC, and the $p$ value by Wilcoxon test was 0.059 .

\subsection{Degree of Necrosis at Explants}

First group: 247 patients with no HCC at explant (blue legend).

Second group: 35 patients with HCC at explant without tumor necrosis (green legend).

Third group: 20 patients with $\mathrm{HCC}$ at explants with only partial necrosis (yellow legend).

Fourth group: 26 patients with HCC at explants showing total necrosis (purple legend).

Figure 5 shows a Kaplan Meier survival analysis comparing the actuarial survival in the 4 groups; the $p$ value by Wilcoxon test was 0.753 , which was statistically insignificant.

\subsection{Cox Regression Multivariate Analysis}

A Cox Regression multivariate analysis was run on the 328 (2001-2007), this is shown in the Table 1.

Pre-OLT MELD was found to be statistically significant as a predictor of survival on Cox Regression multivariate analysis with a $p$ value of 0.023 .

\section{Discussion}

Most of our HCC patients were transplanted in the

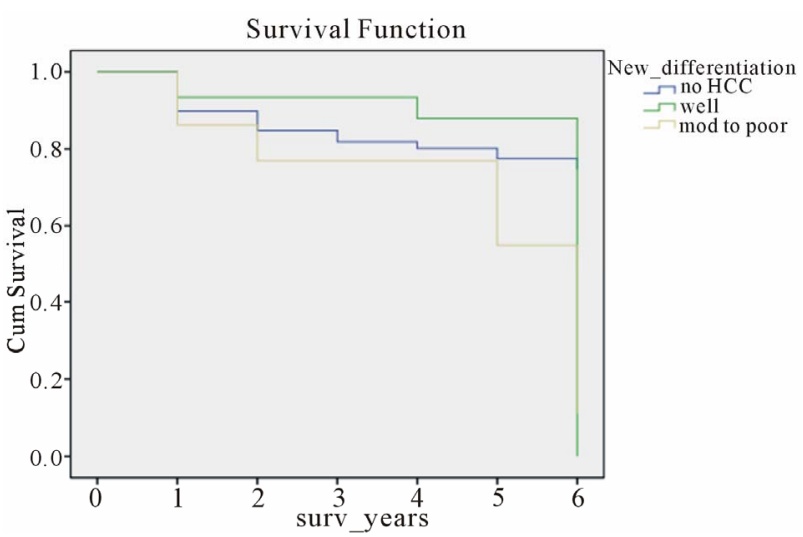

Figure 4. Kaplan Meier survival analysis comparing the actuarial survival according to degree of differentiation at explant pathology (non HCC, poorly differentiated and moderately-well differentiated). 
Table 1. Cox Regression multivariate analysis for 328 patients.

\begin{tabular}{|c|c|c|c|c|c|c|c|c|c|}
\hline & & $\mathrm{B}$ & SE & Wald & $\mathrm{df}$ & Sig. & $\operatorname{Exp}(B)$ & \multicolumn{2}{|c|}{$95.0 \% \mathrm{CI}$ for $\operatorname{Exp}(\mathrm{B})$} \\
\hline & & Lower & Upper & Lower & Upper & Lower & Upper & Lower & Upper \\
\hline \multirow[t]{11}{*}{ Step 1} & New_differentiation & & & 1.145 & 2 & 0.564 & & & \\
\hline & New_differentiation(1) & -0.329 & 0.898 & 0.135 & 1 & 0.714 & 0.719 & 0.124 & 4.183 \\
\hline & New_differentiation(2) & -0.971 & 0.908 & 1.144 & 1 & 0.285 & 0.379 & 0.064 & 2.244 \\
\hline & Milan_score & & & 0.556 & 2 & 0.757 & & & \\
\hline & Milan_score(1) & -0.381 & 1.468 & 0.067 & 1 & 0.795 & 0.683 & 0.038 & 12.128 \\
\hline & Milan_score(2) & -0.684 & 0.918 & 0.554 & 1 & 0.457 & 0.505 & 0.083 & 3.054 \\
\hline & Necrosis & & & 1.212 & 3 & 0.750 & & & \\
\hline & Necrosis(1) & -1.842 & 2.056 & 0.802 & 1 & 0.370 & 0.159 & 0.003 & 8.922 \\
\hline & Necrosis(2) & -0.868 & 1.051 & 0.681 & 1 & 0.409 & 0.420 & 0.054 & 3.295 \\
\hline & Necrosis(3) & -1.152 & 1.106 & 1.085 & 1 & 0.298 & 0.316 & 0.036 & 2.760 \\
\hline & preOLT_MELD & 0.059 & 0.025 & 5.601 & 1 & 0.018 & 1.061 & 1.010 & 1.115 \\
\hline \multirow[t]{8}{*}{ Step 2} & New_differentiation & & & 1.627 & 2 & 0.443 & & & \\
\hline & New_differentiation(1) & -0.499 & 0.760 & 0.432 & 1 & 0.511 & 0.607 & 0.137 & 2.693 \\
\hline & New_differentiation(2) & -1.085 & 0.887 & 1.496 & 1 & 0.221 & 0.338 & 0.059 & 1.922 \\
\hline & Necrosis & & & 0.933 & 3 & 0.818 & & & \\
\hline & Necrosis(1) & -1.397 & 1.709 & 0.668 & 1 & 0.414 & 0.247 & 0.009 & 7.048 \\
\hline & Necrosis(2) & -0.856 & 1.036 & 0.683 & 1 & 0.408 & 0.425 & 0.056 & 3.234 \\
\hline & Necrosis(3) & -0.934 & 1.052 & 0.789 & 1 & 0.375 & 0.393 & 0.050 & 3.088 \\
\hline & preOLT_MELD & 0.057 & 0.024 & 5.488 & 1 & 0.019 & 1.059 & 1.009 & 1.110 \\
\hline \multirow[t]{4}{*}{ Step 3} & New_differentiation & & & 1.000 & 2 & 0.607 & & & \\
\hline & New_differentiation(1) & -0.551 & 0.657 & 0.704 & 1 & 0.401 & 0.576 & 0.159 & 2.089 \\
\hline & New_differentiation(2) & -0.580 & 0.648 & 0.802 & 1 & 0.370 & 0.560 & 0.157 & 1.992 \\
\hline & preOLT_MELD & 0.057 & 0.025 & 5.339 & 1 & 0.021 & 1.058 & 1.009 & 1.111 \\
\hline Step 4 & preOLT_MELD & 0.053 & 0.023 & 5.188 & 1 & 0.023 & 1.055 & 1.007 & 1.104 \\
\hline
\end{tabular}

Only Pre-OLT MELD was found to be statistically significant as a predictor of survival on Cox Regression multivariate analysis with a $p$ value of 0.023 .

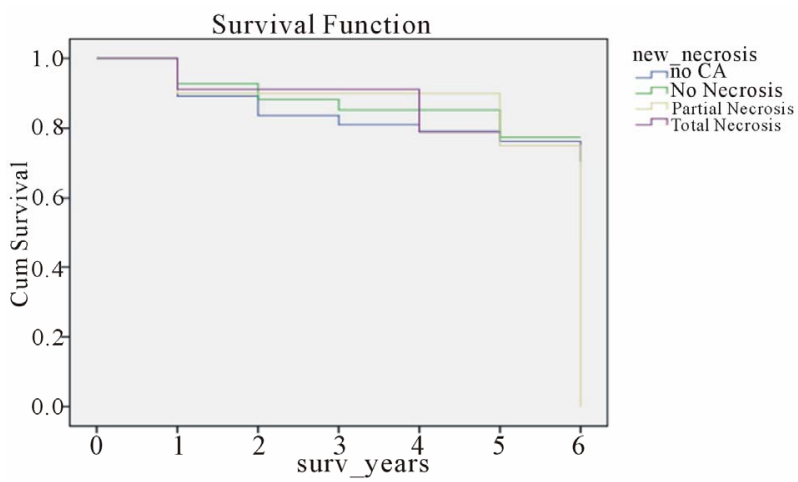

Figure 5. Kaplan Meier survival analysis comparing the actuarial survival according to necrosis at explant pathology (non HCC, no necrosis, partial necrosis, and total necrosis).

MELD era. Those patients are typically transplanted at significantly lower medical MELD scores compared to patients with end stage liver disease without HCC; and the reason for this is to decrease the possibility of $\mathrm{HCC}$ patients to be delisted because of tumor progression; accordingly, the sickest patients are characterized by high mortality both on the waiting list and after liver transplantation. Patients with HCC are transplanted in better condition compared to patients without $\mathrm{HCC}$; thus a similar survival is expected.

Many HCC patients received locoregional therapy as a bridge to OLT to minimize the probability of dropping out from the list due to tumor progression. Typically, locoregional therapy was anticipated once the diagnosis of HCC was established based on characteristic findings on imaging, even for tumors of $2 \mathrm{~cm}$ in diameter or less. The vast majority of our patients had TACE pre-OLT and a minority had RFA with or without TACE.

In our database, we found:

- The whole HCC cohort had no survival disadvantage compared to those without HCC.

- Pre-OLT locoregional therapy did not show any survival advantage, though it led to more necrosis of the tumor at the explant for those who had RFA and significant reduction in tumor size for those who had TACE.

This agrees with the findings recently shown by Amer M. et al., who stated that pre-OLT TACE of $\leq 3 \mathrm{~cm}$ tu- 
mor size did not influence survival post-OLT in patients within Milan criteria [30] compared to those who did not have TACE.

However, these findings were not shown to be significant on univariate analysis, probably because the sample size was too small. Accordingly, we do not have enough numbers of patients who survived long enough to show any statistically significant difference.

\section{Tumor burden and biological behavior:}

We assumed that those having well differentiated HCC might have a better outcome than those having poorly or moderately differentiated HCC. This was based on a different biological behavior and a tendency to metastasize.

In the univariate analysis, those who had complete necrosis by locoregional therapy at explant were not included in this analysis, because the pathologist could not determine the degree of differentiation. The group having well differentiated HCC had a trend for better survival. This variable needs to be studied on a larger number of patients with longer follow-up periods using death from metastatic HCC as an end point, excluding all other causes of mortality to minimize confounding factors. As previously stated in this context, identification of the degree of differentiation of $\mathrm{HCC}$ at the explant is liable to interpersonal and even intrapersonal variations. This is a potential weakness in all retrospective studies having explant specimens examined by more than one pathologist over a relatively long duration of time.

When we tested those having complete necrosis at explant independently in univariate analysis having the same assumption of probable better survival by decreasing tumor burden, we did not find any statistically significant difference. There was no way to make certain whether all of this necrotic tissue was tumor tissue, or some of the surrounding liver tissue which was accidentally targeted by less selective locoregional techniques.

\section{REFERENCES}

[1] M. Shimoda, R. M. Ghobrial, I. C. Carmody, et al., "Predictors of Survival after Liver Transplantation for Hepatocellualr Carcinoma Associated with Hepatitis C," Liver Transplantation, Vol. 10, No. 12, 2004, pp. 1478-1486. http://dx.doi.org/10.1002/lt.20303

[2] B. Ringe, R. Pichlmayr, C. Wittekind, et al., "Surgical Treatment of Hepatocellular Carcinoma: Experience with Liver Resection and Transplantation in 198 Patients," World Journal of Surgery, Vol. 15, No. 2, 1991, pp. 270285. http://dx.doi.org/10.1007/BF01659064

[3] S. Iwatsuki, T. E. Starzl, D. G. Sheahan, et al., "Hepatic Resection versus Transplantation for Hepatocellular Carcinoma," Annals of Surgery, Vol. 214, No. 3, 1991, pp. 221-228.

http://dx.doi.org/10.1097/00000658-199109000-00005

[4] H. Bismuth, L. Chiche, R. Adam, et al., "Surgical Treat- ment of Hepatocellular Carcinoma in Cirrhosis: Liver Resection or Transplantation?" Transplantation Proceedings, Vol. 25, No. 1, 1993, pp. 1066-1067.

[5] Y. Muto, H. Moriwaki, M. Ninomaya, et al., "Prevention of Second Primary Tumors by an Acyclic Retinoid, Polyprenoic Acid, in Patients with Hepatocellular Carcinoma," The New England Journal of Medicine, Vol. 334, No. 24, 1996, pp. 1561-1567.

http://dx.doi.org/10.1056/NEJM199606133342402

[6] W. Y. Lau, T. W. Leung, S. K. Ho, et al., “Adjuvant intra-Arterial Iodine-131-Labelled Lipiodol for Respectable hepatocellular Carcinoma: A Prospective Randomized Trial," Lancet, Vol. 353, No. 9155, 1999, pp. 797-801. http://dx.doi.org/10.1016/S0140-6736(98)06475-7

[7] J. M. Llovet, J. Fuster and J. Bruix, "Intention-to-Treat Analysis of Surgical Treatment for Early Carcinoma: Resection versus Transplantation," Hepatology, Vol. 30, No. 6, 1999, pp. 1434-1440. http://dx.doi.org/10.1002/hep.510300629

[8] Z. Y. Tang, Y. Q. Yu and X. D. Zhou, "Evolution of Surgery in the Treatment of Hepatocellular Carcinoma from the 1950s to the 1990s," Seminars in Surgical Oncology, Vol. 9, No. 4, 1993, pp. 293-297.

http://dx.doi.org/10.1002/ssu.2980090403

[9] M. Makuuchi, "Surgical Management for Hepatocellular Carcinoma," In: V. Arroyo, J. Bosch and J. Rodēs, Eds., Treatments in Hepatology, Barcelona, Masson, Vol. 1995, pp. 341-352.

[10] The Liver Cancer Study Group of Japan, "Predictive Factors for Long Term Prognosis after Partial Hepatectomy for Patients with Hepatocellular Carcinoma in Japan," Cancer, Vol. 74, No. 10, 1994, pp. 2772-2780.

http://dx.doi.org/10.1002/1097-0142(19941115)74:10<27 72::AID-CNCR2820741006>3.0.CO;2-V

[11] B. Ringe, R. Pichlmyar, C. Wittekind, et al., "Surgical Treatment of Hepatocellular Carcinoma: Experience with Liver Resection and Transplantation in 198 Patients," World Journal of Surgery, Vol. 15, No. 2, 1991, pp. 270-285. http://dx.doi.org/10.1007/BF01659064

[12] P. Moreno, E. Jaurrieta, J. Figueras, et al., "Orthotopic Liver Transplantation: Treatment of Choice in Cirrhotic Patients with Hepatocellular Carcinoma?" Transplantation Proceedings, Vol. 27, No. 4, 1995, pp. 2296-2298.

[13] H. Bismuth, L. Chiche, R. Adam, et al., "Liver Resection versus Transplantation for Hepatocellular Carcinoma in Cirrhotic Patients," Annals of Surgery, Vol. 218, No. 2, 1993, pp. 145-151. http://dx.doi.org/10.1097/00000658-199308000-00005

[14] D. G. Farmer, M. H. Rosove, A. Shaked, et al., "Current Treatment Modalities for Hepatocellular Carcinoma," Annals of Surgery, Vol. 219, No. 3, 1994, pp. 236-247. http://dx.doi.org/10.1097/00000658-199403000-00003

[15] H. Bismuth, P. E. Majno and R. Adam, "Liver Transplantation for Hepatocelluar Carcinoma," Seminars in Liver Disease, Vol. 19, No. 3, 1999, pp. 311-322. http://dx.doi.org/10.1055/s-2007-1007120

[16] G. B. Kliontmalm, "Liver Transplantation for Hepatocellular Carcinoma: A Registry Report of the Impact of Tu- 
mor Characteristics on Outcome," Annals of Surgery, Vol. 228, No. 4, 1998, pp. 479-490. http://dx.doi.org/10.1097/00000658-199810000-00005

[17] I. Penn, "Hepatic Transplantation for Primary and Metastatic Cancers of the Liver," Surgery, Vol. 110, No. 4, 1991, pp. 726-734.

[18] I. Yokoyama, S. Todo, S. Iwatsuki, et al., "Liver Transplantation in the Treatment of Primary Liver Cancer," Hepatogastroenterology, Vol. 37, No. 2, 1990, pp. 188193.

[19] J. G. O’Grady, R. J. Polson, K. Rolles, et al., “Liver Transplantion for Malignant Disease. Results in 93 consecutive patients," Annals of Surgery, Vol. 207, No. 4, 1988, pp. 373-379. http://dx.doi.org/10.1097/00000658-198804000-00002

[20] H. Matsunami, Y. Shimizu, S. V. Lynch, et al., "Liver Transplantation as a Therapeutic Option for Hepatocellular Carcinoma," Oncology, Vol. 62, Suppl. 1, 2002, pp. 82-86. http://dx.doi.org/10.1159/000048281

[21] V. Mazzaferro, E. Regalia, R. Doci, et al., "Liver Transplantation for the Treatment of Small Hepatocellular Carcinomas in Patients with Cirrhosis," The New England Journal of Medicine, Vol. 334, No. 11, 1996, pp. 693-699. http://dx.doi.org/10.1056/NEJM199603143341104

[22] F. Y. Yao, L. Ferrell, N. M. Bass, et al., "Liver Transplantation for Hepatocellular Carcinoma: Expansion of the Tumor Size Limits Does Not Adversely Impact Survival," Hepatology, Vol. 33, No. 6, 2001, pp. 1394-1403. http://dx.doi.org/10.1053/jhep.2001.24563

[23] D. Y. Kim, M. S. Choi, J. H. Lee, et al., "Milan Criteria Are Useful Predictors for Favorable Outcomes in Hepatocellular Carcinoma Patients Undergoing Liver Transplantation after Chemoembolization," World Journal of Gastroenterology, Vol. 12, No. 43, 2006, pp. 6992-6997.
[24] “United Network for Organ Sharing,” 2002. http://www.unos.org

[25] F. Y. Yao, N. M. Bass, B. Nikolai, et al., "Liver Transplantation for Hepatocelluar Carcinoma: Analysis of Survival According to the Intention-to-Treat Principle and Dropout from the Waiting List," Liver Transplantation, Vol. 8, No. 10, 2002, pp. 873-883. http://dx.doi.org/10.1053/jlts.2002.34923

[26] I. Yokoyama, B. Carr, H. Saitu, et al., "Accelerated Growth Rate of Recurrent Hepatocellular Carcinoma after Liver Transplantation," Cancer, Vol. 68, No. 10, 1991, pp. 2095-2100.

http://dx.doi.org/10.1002/1097-0142(19911115)68:10<20 95::AID-CNCR2820681002>3.0.CO $2-Y$

[27] I. Penn, "The Effect of Immunosuppression on Pre-Existing Cancers," Transplantation, Vol. 55, No. 4, 1993, pp. 742-747.

http://dx.doi.org/10.1097/00007890-199304000-00011

[28] C. E. Freise, L. Ferrell, T. Liu, et al., "Effect of Systemic Cyclosporin on Tumor Recurrence after Liver Transplantation in a Model of Hepatocellular Carcinoma," Transplantation, Vol. 67, No. 4, 1999, pp. 510-513. http://dx.doi.org/10.1097/00007890-199902270-00003

[29] V. Marco, B. Roberto, C. Alessandro, et al., "Low Recurrence Rate of Hepatocellular Carcinoma after Liver Transplantation: Better Patient Selection or Lower Immunosuppression?" Transplantation, Vol. 74, No. 12, 2002, pp. 1746-1751.

[30] M. Amer, M. Yousri, F. Barakat, et al., "Pretransplant Chemoembolization for Hepatocellular Carcinoma $\leq \mathrm{cm}$ Does Not Influence the Outcome after Liver Transplantation," 58th annual meeting of the AASLD, November 2007. 